\title{
On the Maximum Number of Translates in a Point Set
}

\author{
Bernardo M. Ábrego • \\ Silvia Fernández-Merchant • Bernardo Llano
}

Received: 17 January 2008 / Revised: 10 June 2008 / Accepted: 5 September 2008 /

Published online: 24 September 2008

(C) Springer Science+Business Media, LLC 2008

\begin{abstract}
Given a finite set $P \subseteq \mathbb{R}^{d}$, called a pattern, $t_{P}(n)$ denotes the maximum number of translated copies of $P$ determined by $n$ points in $\mathbb{R}^{d}$. We give the exact value of $t_{P}(n)$ when $P$ is a rational simplex, that is, the points of $P$ are rationally affinely independent. In this case, we prove that $t_{P}(n)=n-m_{r}(n)$, where $r$ is the rational affine dimension of $P$, and $m_{r}(n)$ is the $r$-Kruskal-Macaulay function. We note that almost all patterns in $\mathbb{R}^{d}$ are rational simplices. The function $t_{P}(n)$ is also determined exactly when $|P| \leq 3$ or when $P$ has rational affine dimension one and $n$ is large enough. We establish the equivalence of finding $t_{P}(n)$ and the maximum number $s_{R}(n)$ of scaled copies of a suitable pattern $R \subseteq \mathbb{R}^{+}$determined by $n$ positive reals. As a consequence, we show that $s_{A_{k}}(n)=n-\Theta\left(n^{1-1 / \pi(k)}\right)$, where $A_{k}=\{1,2, \ldots, k\}$ is an arithmetic progression of size $k$, and $\pi(k)$ is the number of primes less than or equal to $k$.
\end{abstract}

Research partially supported by PIFI 3.1-3.2, UAM-I-CA-53 Análisis Aplicado, when the first two authors visited B. Llano.

B.M. Ábrego $(\varangle) \cdot$ S. Fernández-Merchant

Department of Mathematics, California State University, Northridge, 18111 Nordhoff St, Northridge, CA 91330-8313, USA

e-mail: bernardo.abrego@csun.edu

B.M. Ábrego $\cdot$ S. Fernández-Merchant

Centro de Investigación en Matemáticas, A.C., Jalisco S/N Colonia Valenciana, 36240 Guanajuato, GTO, Mexico

S. Fernández-Merchant

e-mail: silvia.fernandez@csun.edu

B. Llano

Departamento de Matemáticas, Universidad Autónoma Metropolitana, Iztapalapa San Rafael Atlixco 186, Colonia Vicentina, 09340, México, D.F., Mexico

e-mail: 1lano@xanum.uam.mx 
Keywords Pattern · Geometric pattern · Translates · Translated copies · Scaled copies $\cdot$ Kruskal-Macaulay $\cdot$ Rational dimension $\cdot$ Rational simplex

\section{Introduction}

Some of the most natural and important problems in Combinatorial Geometry are those concerning repeated configurations and pattern recognition. A typical question in the area is the following:

What is the maximum number of occurrences of a pattern among all subsets of an $n$-point set in the $d$-dimensional space?

Here an occurrence is determined by a class of equivalence under a fixed group of geometric transformations: translations, homotheties, congruences, similarities, and affinities. Many of these problems were first asked by Erdős and Purdy [10, 11]. For instance, when $d=2$, the geometric group are congruences, and the pattern consists of the endpoints of a unit segment; we obtain Erdős' famous problem [9] (still wide open) about the maximum number of unit segments that can be determined by a set of $n$ points in the plane.

In general, determining the exact maxima for these problems is hard. The only nontrivial instances where an exact formula is known are due to Brass [5] and van Wamelen [17], who worked with unit segments (congruences) in $\mathbb{R}^{4}$, and to Swanepoel [16], who very recently accomplished the impressive task of obtaining exact formulas for the maximum number of unit segments determined by $n$ points in $\mathbb{R}^{2 d}$, for $d \geq 3$ and for every $n$ large enough. He also found structural properties for the optimal sets in odd dimensions $d$.

In spite of this, there are very few known asymptotic formulae. In the best case, one hopes to determine the order of magnitude for the corresponding maximum, and even this is only known for few particular patterns and geometric transformations. Elekes and Erdôs [8] found the right order of magnitude for algebraic patterns in $\mathbb{R}^{d}$ under homotheties and in the plane under similarities. For arbitrary patterns, their upper bounds are almost matched by their constructions. Continuing with similarities in the plane, Laczkovich and Rusza [13] classified the patterns that can have a quadratic number of similar copies (the maximum possible), and Ábrego, Elekes, and Fernández-Merchant [1] found structural properties on those point-sets having a quadratic number of occurrences. The problem of similarities when $d \geq 3$ gets harder. Even for the simplest case of triangles in 3-space, we do not know the right order of magnitude.

It is worth mentioning that the algorithmic aspect of these combinatorial questions is motivated by real-life necessities: finding patterns among the huge amounts of data obtained by scanners, digital cameras, electron microscopes, telescopes, etc. The reader is encouraged to see [4, 6, 7], and [15] for further references and the current status of both the combinatorial and the algorithmic problems.

In this paper, we consider the problem of finding the maximum number of translated copies of a pattern that can occur among $n$ points in $d$-dimensional space. More 


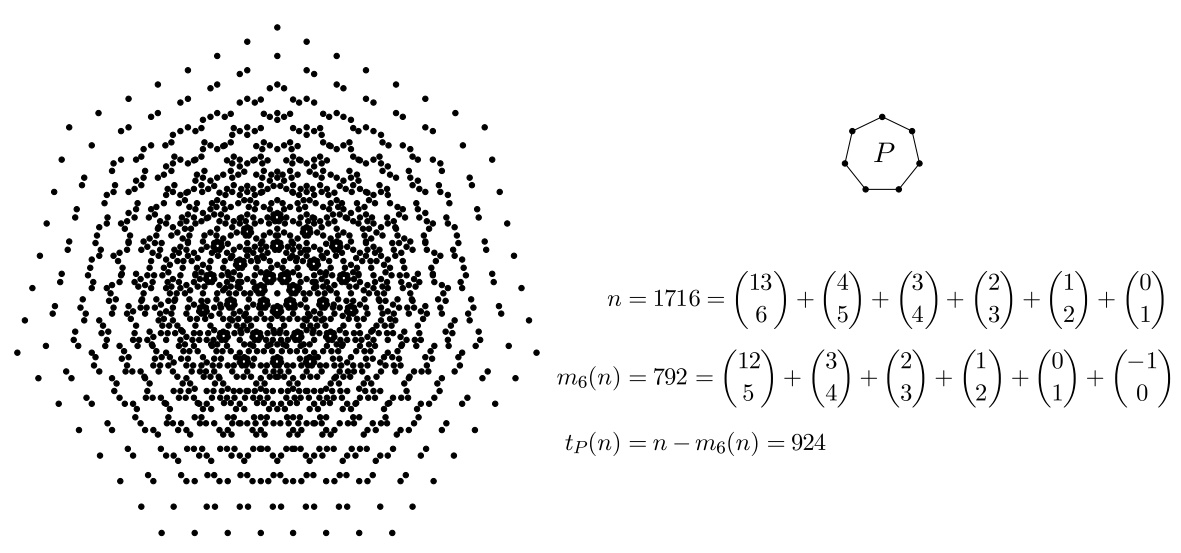

Fig. 1 Optimal set with $n=1716$ points and the maximum number of translates of $P$, the regular heptagon

precisely, let $P \subseteq \mathbb{R}^{d}$ be a fixed finite subset of the $d$-dimensional real space. We refer to $P$ as a pattern. For any finite set $X \subseteq \mathbb{R}^{d}$, let $t_{P}(X)$ be the number of translated copies, or simply translates, of $P$ contained in $X$. That is,

$$
t_{P}(X)=\mid\left\{P^{\prime} \subseteq X: P^{\prime}=v+P \text { where } v \in \mathbb{R}^{d}\right\} \mid .
$$

We want to determine the largest possible value of $t_{P}(X)$ among all $n$-point sets $X \subseteq \mathbb{R}^{d}$. We denote this by

$$
t_{P}(n)=\max \left\{t_{P}(X): X \subseteq \mathbb{R}^{d},|X|=n\right\} .
$$

This problem was previously considered by Brass [4]. It was also presented as a prototype, by Pach and Sharir, for the many problems about repeated configurations and pattern recognition treated in [15]. Brass defined the rational affine dimension, $\operatorname{dim}_{\mathbb{Q}} P$, of a pattern $P \subseteq \mathbb{R}^{d}$ as the dimension of the affine space generated by $P$. This is precisely the rational linear dimension of the set generated by $P+(-P)=$ $\left\{p_{1}-p_{2}: p_{1}, p_{2} \in P\right\}$, i.e., the size of the smallest set $B$ such that any element of $P+(-P)$ is a linear combination with rational coefficients of elements of $B$. Note that the rational linear dimension of a set may be larger than its rational affine dimension, but when $P$ contains the origin, the two dimensions coincide. Brass found the asymptotic value of $t_{P}(n)$ in terms of the rational affine dimension $r$ of $P$. He proved that

$$
t_{P}(n)=n-\Theta\left(n^{1-1 / r}\right) .
$$

Our main result gives the exact value of $t_{P}(n)$ when the pattern $P$ is a rational simplex, that is, $|P|=\operatorname{dim}_{\mathbb{Q}} P+1$. We remark that almost all patterns $P$ in $\mathbb{R}^{d}$ are rational simplices. If we were to select a pattern at random (with a fixed number of points) from a compact subset of $\mathbb{R}^{d}$ with positive finite measure, then the pattern would be a rational simplex with probability one. Some interesting patterns are also rational simplices. For instance, a regular polygon with prime number of sides (Fig. 1), a regular pentagonal pyramid (Fig. 2), or the set $\{0,1, \sqrt{2}, \sqrt{3}, e\}$. 


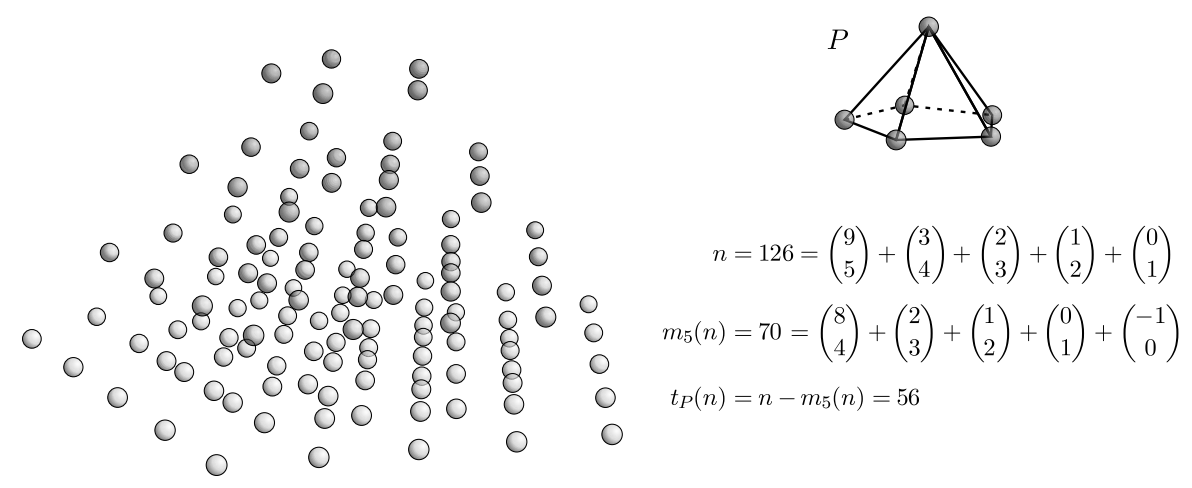

Fig. 2 Optimal set with $n=126$ points and the maximum number of translates of $P$, the pentagonal pyramid

To present our explicit formula we need some definitions from finite set theory. Given positive integers $r$ and $n$, there is a unique way of writing $n$ as

$$
n=\left(\begin{array}{c}
n_{r} \\
r
\end{array}\right)+\left(\begin{array}{c}
n_{r-1} \\
r-1
\end{array}\right)+\cdots+\left(\begin{array}{c}
n_{2} \\
2
\end{array}\right)+\left(\begin{array}{c}
n_{1} \\
1
\end{array}\right)
$$

so that $0 \leq n_{1}<n_{2}<\cdots<n_{r-1}<n_{r}$ are integers. Using this representation, called the $r$-binomial representation of $n$, the $r$-Kruskal-Macaulay function, is defined as

$$
m_{r}(n)=\left(\begin{array}{c}
n_{r}-1 \\
r-1
\end{array}\right)+\left(\begin{array}{c}
n_{r-1}-1 \\
r-2
\end{array}\right)+\cdots+\left(\begin{array}{c}
n_{2}-1 \\
1
\end{array}\right)+\left(\begin{array}{c}
n_{1}-1 \\
0
\end{array}\right) .
$$

(See $[3,12]$ for details.) Our main result, proved in Sect. 2, is the following:

Theorem 1 If $P$ is a rational simplex with $\operatorname{dim}_{\mathbb{Q}}(P)=r$, then

$$
t_{P}(n)=n-m_{r}(n) .
$$

Since any pattern $P$ with rational affine dimension $r$ contains an $r$-dimensional rational simplex, $t_{P}(n) \leq n-m_{r}(n) \leq n-\frac{r}{(r !)^{1 / r}} n^{1-1 / r}+O\left(n^{1-2 / r}\right)$. Brass also used the $r$-dimensional rational simplex to find an upper bound for $t_{P}(n)$. Implicit in his proof [4] is the bound $t_{P}(n) \leq n-\sqrt{\pi} /\left(2 \Gamma(r / 2+1)^{1 / r}\right) n^{1-1 / r}$, where $\Gamma$ is Euler's Gamma Function. Our result improves this inequality and gives a tight lower bound for the leading coefficient of the error term. As a way of comparing, as $r \rightarrow \infty$, Brass' coefficient goes to zero, whereas ours goes to $e$.

We also consider the following problem about maximizing scaled copies of a pattern. Let $R$ be a fixed finite set of positive reals. Again, we refer to $R$ as a (real) pattern. For any finite set $Y \subseteq \mathbb{R}^{+}$, let $s_{R}(Y)$ be the number of scaled copies of $R$ contained in $Y$. That is,

$$
s_{R}(Y)=\mid\left\{R^{\prime} \subseteq Y: R^{\prime}=\lambda R \text { for some } \lambda \in \mathbb{R}^{+}\right\} \mid .
$$


We want to determine the value of $s_{R}(n)=\max \left\{s_{R}(Y): Y \subseteq \mathbb{R}^{+},|Y|=n\right\}$.

The following result shows the equivalence of maximizing the number of scaled copies of a real pattern and maximizing the number of translates of an equivalent pattern. Its proof, included in Sect. 3, provides explicit ways of constructing a pattern $P$ in $\mathbb{R}^{d}$ from a pattern $R$ of positive reals, and vice versa. Before stating the theorem, we introduce some terminology from abstract algebra. If $R$ is a finite set in $\mathbb{R}^{+}$, we denote by $\langle R\rangle$ the smallest multiplicative subgroup of $\mathbb{R}^{+}$containing $R$. By construction $\langle R\rangle$ is a finitely generated free abelian group. That is, there is a finite subset of $\langle R\rangle$, called a basis, which satisfies that every element of the group can be written in one and only one way as a finite product of integer powers of the elements in the basis. In general, a free abelian group has many different bases, but all bases have the same cardinality, and this cardinality is called the rank of the group.

Theorem 2 Let $n \in \mathbb{N}$. For every pattern $R \subseteq \mathbb{R}^{+}$, there exists a pattern $P \subseteq \mathbb{Z}^{d}$, where $d$ is the rank of $R$, such that

$$
s_{R}(n)=t_{P}(n)
$$

Reciprocally, for every dimension $d$ and every pattern $P \subseteq \mathbb{R}^{d}$, there is a pattern $R \subseteq \mathbb{N}$ satisfying $s_{R}(n)=t_{P}(n)$. Moreover, $|R|=|P|$, and if $1 \in R$, or equivalently if $0 \in P$, then the rank of $R$ and the rational affine dimension of $P$ coincide.

Before proving this theorem, at the beginning of Sect. 3, we present some of its applications to the number of scaled copies of an arithmetic progression. Let $A_{k}=$ $\{1,2,3, \ldots, k\}$; if $k \leq 4$, we determine precisely $s_{A_{k}}(n)$ for every $n$. We also show the following:

Theorem 3 If $k \in \mathbb{N}$, then $s_{A_{k}}(n)=n-\Theta\left(n^{1-1 / \pi(k)}\right)$, where $\pi(k)$ is the number of primes not exceeding $k$.

In Sect. 4, we concentrate on the 1-dimensional (rational affine) patterns. We essentially solve the problem. We give the exact value of $t_{P}(n)$ for $n$ large enough and any pattern $P$ with $|P| \geq 3$ by showing the following:

Theorem 4 Let $P=\left\{0, a_{1}, a_{2}, \ldots, a_{k+1}\right\} \subseteq \mathbb{Z}$ with $k \geq 1,0<a_{1}<a_{2}<\cdots<$ $a_{k+1}=M$, and such that $\operatorname{gcd}\left\{a_{i}\right\}_{i=1}^{k+1}=1$. Then

$$
t_{P}(n)=n-M \quad \text { for every } n \geq M(M-k) .
$$

We give the exact values of $t_{P}(n)$ for all $n$ when $|P| \leq 3: t_{P}(n)=n$ for $|P|=1$ is trivial, $t_{P}(n)=n-1$ if $|P|=2$ is a consequence of Theorem 1 , and for $|P|=3$, we show the following:

Theorem 5 Let $P=\{0, a, b\} \subseteq \mathbb{Z}$ with $0<a<b$ and $\operatorname{gcd}(a, b)=1$. Then

$$
t_{P}(n)= \begin{cases}n-\left\lceil\sqrt{2 n+\frac{1}{4}}-\frac{1}{2}\right\rceil & \text { if } n \leq\left(\begin{array}{c}
b \\
2
\end{array}\right), \\
n-b & \text { if } n>\left(\begin{array}{l}
b \\
2
\end{array}\right) .\end{cases}
$$


Finally, in Sect. 5, we study 2-dimensional (rational affine) patterns with four points. We determine $t_{\square}(n)$ and $t_{L}(n)$, where $\square$ represents the pattern in $\mathbb{R}^{2}$ consisting of the four vertices of a square, and $L=\{(0,0),(1,0),(2,0),(0,1)\}$. We also present some conjectures about the exact value of $t_{P}(n)$ for every set $P$ with $|P|=4$.

In this paper, we denote by $\mathbb{N}$ the set of positive integers and by $\mathbb{N}^{*}=\mathbb{N} \cup\{0\}$ the set of nonnegative integers.

\section{Proof of Theorem 1: Translates of a Rational Simplex}

\subsection{Preliminaries and Discretization}

Let $P$ be a finite set in $\mathbb{R}^{d}$. By translating $P$ if necessary, we can assume that $0 \in P$. Let $\langle P\rangle_{+}$be the additive subgroup of $\mathbb{R}^{d}$ generated by $P$ and $\left\{v_{1}, v_{2}, \ldots, v_{r}\right\}$ a basis of $\langle P\rangle_{+}$. Then $\langle P\rangle_{+}=\left\{k_{1} v_{1}+k_{2} v_{2}+\cdots+k_{r} v_{r}: k_{i} \in \mathbb{Z}\right\}$, and, since $0 \in P, r$ is the rational affine dimension of $P$. Note that any translate $v+P$ of $P$ is contained in a single equivalence class $v+\langle P\rangle_{+} \in \mathbb{R}^{d} /\langle P\rangle_{+}$. The following lemma restricts the maximum for the function $t_{P}(n)$ to $\langle P\rangle_{+}$.

Lemma 1 For any finite set $P \subseteq \mathbb{R}^{d}$,

$$
t_{P}(n)=\max \left\{t_{P}(X): X \subseteq\langle P\rangle_{+},|X|=n\right\} .
$$

Proof Take $X \subseteq \mathbb{R}^{d}$ and assume that $X \supseteq X_{1} \cup X_{2}$, where $X_{1}$ and $X_{2}$ are contained in different classes $u_{1}+\langle P\rangle_{+}$and $u_{2}+\langle P\rangle_{+}$, respectively. For $k \in \mathbb{Z}$ large enough, the set $X_{2}^{\prime}=\left(u_{1}-u_{2}+k v_{1}\right)+X_{2} \subseteq u_{1}+\langle P\rangle_{+}$has no elements in common with $X$. Also $X_{2}^{\prime}$ has as many translates of $P$ as $X_{2}$. Replacing $X_{2}$ by $X_{2}^{\prime}$ in $X$, we obtain a set intersecting a fewer number of equivalence classes in $\mathbb{R}^{d} /\langle P\rangle_{+}$and with at least as many translates of $P$ as $X$. Now, if $X$ is contained in a single class $u_{1}+\langle P\rangle_{+}$, consider the set $\left(-u_{1}\right)+X \subseteq\langle P\rangle_{+}$. Since this set contains as many translated copies of $P$ as $X$, the identity is satisfied.

\subsection{Reduction to the Standard Simplex and Construction of $B_{r}(n)$}

Let $P_{r} \subseteq \mathbb{R}^{r}$ be the standard $r$-simplex consisting of 0 and the $r$ standard basis vectors $e_{1}, e_{2}, \ldots, e_{r}$. If $P$ is a rational simplex with $\operatorname{dim}_{\mathbb{Q}}(P)=r$, we can assume that $P=\left\{0, v_{1}, v_{2}, \ldots, v_{r}\right\}$ by using a suitable translation. Under the linear transformation (from $\langle P\rangle_{+}$to $\mathbb{Z}^{r}$ ) that sends $v_{i}$ to $e_{i}$, translates of $P$ correspond to translates of $P_{r}$. Thus, by Lemma 1 , we have that

$$
\begin{aligned}
t_{P}(n) & =\max \left\{t_{P}(X): X \subseteq\langle P\rangle_{+},|X|=n\right\} \\
& =\max \left\{t_{P_{r}}(X): X \subseteq \mathbb{Z}^{r},|X|=n\right\} .
\end{aligned}
$$

We first construct an $n$-set $B_{r}(n) \subseteq \mathbb{Z}^{r}$ having at least $n-m_{r}(n)$ translates of $P_{r}$. Then by (3), $t_{P}(n) \geq n-m_{r}(n)$. We construct $B_{r}(n)$ recursively as follows. For every $n \in \mathbb{N}$, set $B_{1}(n)=\{1,2, \ldots, n\}$. For $r \geq 2$, if $n=\left(\begin{array}{c}n_{r} \\ r\end{array}\right)$ for some $n_{r} \in \mathbb{Z}$, let

$$
\begin{aligned}
B_{r}\left(\left(\begin{array}{c}
n_{r} \\
r
\end{array}\right)\right)=\{ & \left(x_{1}, x_{2}, \ldots, x_{r}\right) \in \mathbb{Z}^{r}: x_{i} \geq 0, x_{r} \geq 1, \\
& \text { and } \left.x_{1}+x_{2}+\cdots+x_{r} \leq n_{r}-r+1\right\} .
\end{aligned}
$$



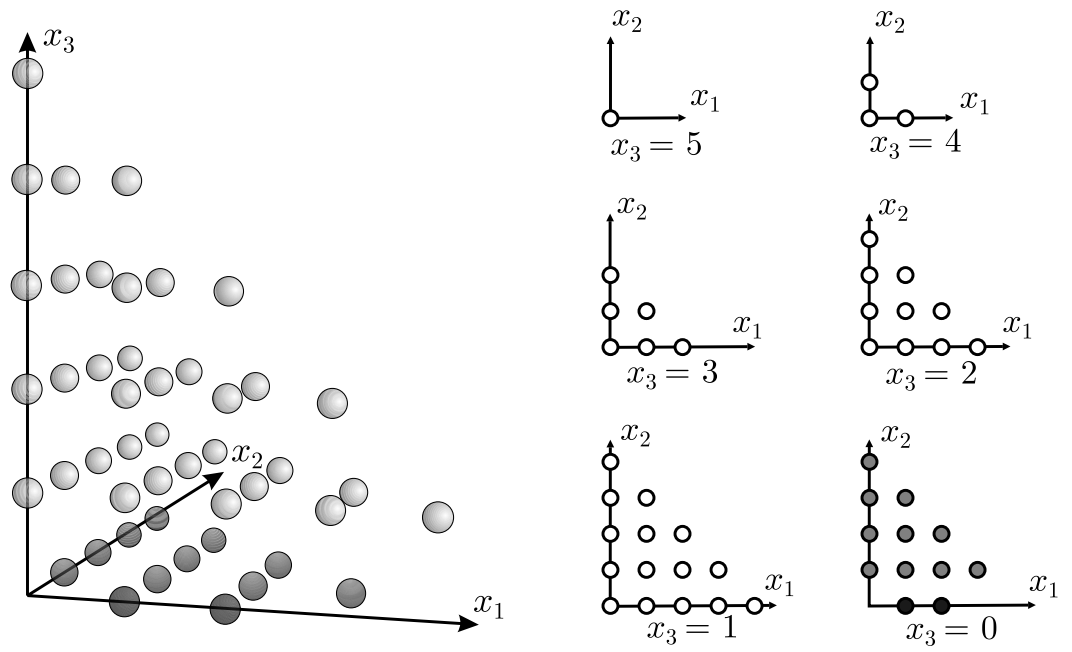

Fig. 3 Construction of $B_{3}(n)$ with $n=\left(\begin{array}{l}7 \\ 3\end{array}\right)+\left(\begin{array}{l}5 \\ 2\end{array}\right)+\left(\begin{array}{l}2 \\ 1\end{array}\right)=47$ points and $n-m_{3}(n)=27$ translates of $P_{3}$

Note that this set has exactly $\left(\begin{array}{c}n_{r} \\ r\end{array}\right)$ points. For all other values of $n$, consider the $r$ binomial representation $n=\left(\begin{array}{c}n_{r} \\ r\end{array}\right)+\left(\begin{array}{c}n_{r-1} \\ r-1\end{array}\right)+\cdots+\left(\begin{array}{c}n_{2} \\ 2\end{array}\right)+\left(\begin{array}{c}n_{1} \\ 1\end{array}\right)$ and let $n^{\prime}=n-\left(\begin{array}{c}n_{r} \\ r\end{array}\right)>0$. Define

$$
B_{r}(n)=B_{r}\left(\left(\begin{array}{c}
n_{r} \\
r
\end{array}\right)\right) \cup\left(B_{r-1}\left(n^{\prime}\right) *\{0\}\right),
$$

where $B_{r-1}\left(n^{\prime}\right) *\{0\}$ consists of the points in $B_{r-1}\left(n^{\prime}\right)$ with an extra coordinate zero appended to them (see Fig. 3). Observe that $B_{r}(n)$ has exactly $n$ points and, by construction, all points $x=\left(x_{1}, x_{2}, \ldots, x_{r}\right)$ satisfy that $x_{1}+x_{2}+\cdots+x_{r} \leq$ $n_{r}-r+1$. (This is clear for $x \in B_{r}\left(\left(\begin{array}{c}n_{r} \\ r\end{array}\right)\right)$, otherwise $x \in B_{r-1}\left(n^{\prime}\right) *\{0\}$ and thus $\left(x_{1}+x_{2}+\cdots+x_{r-1}\right)+x_{r} \leq n_{r-1}-(r-1)+1+0 \leq n_{r}-r+1$ since $n_{r-1}<n_{r}$.

\subsection{The Number of Translates of the Standard Simplex in $B_{r}(n)$}

We prove that $t_{P_{r}}\left(B_{r}(n)\right) \geq n-m_{r}(n)$ by induction on $r$. If $r=1$, then $B_{r}(n)=$ $\{1,2, \ldots, n\}$, which clearly contains $n-1$ copies of $P_{1}=\{0,1\}$, that is, $t_{P_{r}}\left(B_{r}(n)\right)=$ $n-1=n-m_{1}(n)$. Let $r \geq 2$ and assume that the result is true for $r-1$. We bound the number of points $x$ in $B_{r}(n)$ such that $x+P_{r} \subseteq B_{r}(n)$, observing that different points generate different translates of $P_{r}$. We do this first for points in $B_{r}\left(\left(\begin{array}{c}n_{r} \\ r\end{array}\right)\right)$ and then for points in $B_{r-1}\left(n^{\prime}\right) *\{0\}$.

If $x \in B_{r}\left(\left(\begin{array}{c}n_{r} \\ r\end{array}\right)\right)$ and $x_{1}+x_{2}+\cdots+x_{r} \leq n_{r}-r$, then $x+e_{i} \in B_{r}\left(\left(\begin{array}{c}n_{r} \\ r\end{array}\right)\right)$ for $1 \leq i \leq r$. Therefore, $x+P_{r} \subseteq B_{r}\left(\left(\begin{array}{c}n_{r} \\ r\end{array}\right)\right)$, and thus $t_{P_{r}}\left(B_{r}\left(\left(\begin{array}{c}n_{r} \\ r\end{array}\right)\right)\right) \geq\left(\begin{array}{c}n_{r} \\ r\end{array}\right)-\left(\begin{array}{c}n_{r}-1 \\ r-1\end{array}\right)$.

Now, suppose that $x^{\prime}+P_{r-1}$ is a translate of $P_{r-1}$ in $B_{r-1}\left(n^{\prime}\right)$ with $x^{\prime}=$ $\left(x_{1}, x_{2}, \ldots, x_{r-1}\right)$. Then the point $x^{\prime} *\{0\} \in B_{r-1}\left(n^{\prime}\right) *\{0\} \subseteq B_{r}(n)$, and we claim that $x^{\prime} *\{0\}+P_{r} \subseteq B_{r}(n)$. Indeed, for $1 \leq i<r$, we have that $x^{\prime}+e_{i} \in B_{r-1}\left(n^{\prime}\right)$ and then $x^{\prime} *\{0\}+e_{i}=\left(x^{\prime}+e_{i}\right) *\{0\} \in B_{r-1}\left(n^{\prime}\right) *\{0\} \subseteq B_{r}(n)$. In particular, $x_{1}+x_{2}+\cdots+x_{r-1}+1 \leq n_{r}-r+1$, and thus $x^{\prime} *\{0\}+e_{r}=\left(x_{1}, x_{2}, \ldots, x_{r-1}, 1\right) \in$ 
$B_{r}\left(\left(\begin{array}{c}n_{r} \\ r\end{array}\right)\right) \subseteq B_{r}(n)$. All this gives at least $t_{P_{r-1}}\left(n^{\prime}\right)$ translates $x+P_{r}$ in $B_{r}(n)$ with $x \in B_{r-1}\left(n^{\prime}\right) *\{0\}$. Finally, by induction $t_{P_{r-1}}\left(n^{\prime}\right) \geq n^{\prime}-m_{r-1}\left(n^{\prime}\right)$, and thus,

$$
\begin{aligned}
t_{P_{r}}\left(B_{r}(n)\right) & \geq t_{P_{r}}\left(B_{r}\left(\left(\begin{array}{c}
n_{r} \\
r
\end{array}\right)\right)\right)+t_{P_{r-1}}\left(n^{\prime}\right) \\
& \geq\left(\begin{array}{c}
n_{r} \\
r
\end{array}\right)-\left(\begin{array}{c}
n_{r}-1 \\
r-1
\end{array}\right)+n^{\prime}-m_{r-1}\left(n^{\prime}\right)=n-m_{r}(n) .
\end{aligned}
$$

\subsection{Bounding $t_{P}(n)$ Above}

To prove the reverse inequality, $t_{P}(n) \leq n-m_{r}(n)$, we use the following result from [2]. This inequality is tight for every $r$ and infinitely many values of $n$. In [2] the authors use this inequality to give a short proof of Macaulay's theorem (see $[12,14])$.

Lemma 2 If $r \geq 2$ and $0 \leq a<m_{r}(n)$, then

$$
m_{r-1}(a)+m_{r}(n-a) \geq m_{r}(n) .
$$

Moreover, if $n=\left(\begin{array}{l}N \\ r\end{array}\right)$ for some $N \geq r$, then equality in (4) occurs only when $a=0$.

Let $X$ be an $n$-point set in $\mathbb{Z}^{r}$. A point $x \in X$ is called a deficit point with respect to $P_{r}$ if $x+P_{r} \nsubseteq X$. Define $d_{r}(X)$ as the number of deficit points with respect to $P_{r}$ in the set $X$. Clearly $t_{P_{r}}(X)=n-d_{r}(X)$. We prove by induction, on $r$ first and then on $n$, that $d_{r}(X) \geq m_{r}(n)$ for all $n$-point sets $X \subseteq \mathbb{Z}^{r}$. This, together with (3), gives $t_{P}(n)=n-\min \left\{d_{r}(X): X \subseteq \mathbb{Z}^{r},|X|=n\right\} \leq n-m_{r}(n)$.

If $r=1$, then clearly the largest point in $X \subseteq \mathbb{Z}$ is a deficit point, thus $d_{1}(X) \geq$ $1=m_{1}(n)$. Suppose that $r \geq 2$ and that the inequality holds for $r-1$. If $n \leq r$, then the result is trivially true since $d_{r}(X)=n=m_{r}(n)$. Assume that $n \geq r+1$ and that the result holds for values smaller than $n$. By using an appropriate translation, we can assume that the $r$-coordinate of every point in $X$ is greater than zero and that there are some points with $r$-coordinate equal to one. For every $i \geq 1$, let $L_{i}$ be the points in $X$ with $r$-coordinate equal to $i$. Let $l_{i}=\left|L_{i}\right|$ and note that $l_{1} \geq 1$.

First assume that $l_{1} \geq m_{r}(n)$. Observe that every non-deficit point $x$ in $L_{i}$ is bijectively associated to the point $x+e_{r} \in L_{i+1}$. Then there are at least $l_{i}-l_{i+1}$ deficit points in $L_{i}$. Assuming that $u$ is the largest subindex such that $l_{u} \neq 0$, we get $d_{r}(X) \geq \sum_{i=1}^{u}\left(l_{i}-l_{i+1}\right)=l_{1} \geq m_{r}(n)$.

On the other hand, if $l_{1}<m_{r}(n)$, then, by Lemma 2, inequality (4) holds with $a=l_{1}$. This time note that every deficit point with respect to $P_{r-1}$ in $L_{i}$ is also a deficit point with respect to $P_{r}$ in $X$, which means that $d_{r}\left(L_{i}\right) \geq d_{r-1}\left(L_{i}\right)$. Thus $d_{r}(X) \geq d_{r-1}\left(L_{1}\right)+d_{r}\left(X \backslash L_{1}\right)$. By induction on $r, d_{r-1}\left(L_{1}\right) \geq m_{r-1}\left(l_{1}\right)$, and by induction on $n, d_{r}\left(X \backslash L_{1}\right) \geq m_{r}\left(n-l_{1}\right)$. These, together with (4), give $d_{r}(X) \geq m_{r}(n)$.

Remark 1 The problem of classifying the $n$-sets $X$ for which $t_{P_{r}}(X)=t_{P_{r}}(n)$ is open. The first difficulty would be to determine the pairs $(a, n)$ for which we get identity in (4). However, for $n$ of a particular form, we can determine uniqueness for the optimal sets constructed in Sect. 2.2 (in particular the sets shown in Figs. 1 and 2 are unique). 
Proposition 1 If $n=\left(\begin{array}{c}N \\ r\end{array}\right)$, then the set $X=B_{r}(n)$ is the unique $n$-set in $\mathbb{Z}^{r}$ (modulo translations) for which $t_{P_{r}}(X)=t_{P_{r}}(n)$.

Proof According to the proof of Theorem 1, $d_{r}(X)=m_{r}(n)$. If $l_{1}<m_{r}(n)=\left(\begin{array}{c}N-1 \\ r-1\end{array}\right)$, then $d_{r}(X) \geq d_{r-1}\left(L_{1}\right)+d_{r}\left(X \backslash L_{1}\right) \geq m_{r-1}\left(l_{1}\right)+m_{r}\left(n-l_{1}\right)$. Because $l_{1}>0$, it follows, by Lemma 2, that (4) is strict. Thus,

$$
m_{r}(n)=d_{r}(X) \geq m_{r-1}\left(l_{1}\right)+m_{r}\left(n-l_{1}\right)>m_{r}(n),
$$

which is impossible. For the other case, we must have $l_{1} \geq m_{r}(n)$. Then $m_{r}(n)=$ $d_{r}(X) \geq l_{1} \geq m_{r}(n)$, i.e., $l_{1}=m_{r}(n)=\left(\begin{array}{c}N-1 \\ r-1\end{array}\right)$. Further,

$$
\begin{aligned}
m_{r}(n) & =d_{r}(X) \geq d_{r-1}\left(L_{1}\right)+d_{r}\left(X \backslash L_{1}\right) \geq m_{r-1}\left(l_{1}\right)+m_{r}\left(n-l_{1}\right) \\
& \geq m_{r-1}\left(\left(\begin{array}{c}
N-1 \\
r-1
\end{array}\right)\right)+m_{r}\left(\left(\begin{array}{c}
N-1 \\
r
\end{array}\right)\right)=\left(\begin{array}{c}
N-2 \\
r-2
\end{array}\right)+\left(\begin{array}{c}
N-2 \\
r-1
\end{array}\right)=\left(\begin{array}{c}
N-1 \\
r-1
\end{array}\right)=m_{r}(n) .
\end{aligned}
$$

Thus equality must occur everywhere. Then $L_{1}$ is an optimal set for $P_{r-1}$ with $\left(\begin{array}{c}N-1 \\ r-1\end{array}\right)$ points, and $X \backslash L_{1}$ is an optimal set for $P_{r}$ with $\left(\begin{array}{c}N-1 \\ r\end{array}\right)$ points. By induction, $L_{1}$ and $X \backslash L_{1}$ are translates of $B_{r-1}\left(l_{1}\right)$ and $B_{r}\left(n-l_{1}\right)$, the optimal sets constructed in Sect. 2.2. Moreover, to have equality in $d_{r}(X) \geq d_{r-1}\left(L_{1}\right)+d_{r}\left(X \backslash L_{1}\right)$, we need that every non-deficit point in $L_{1}$ is exactly below, in the $x_{r}$ coordinate, a point from $X \backslash L_{1}$. This can only happen if $L_{1}$ is the bottom layer (in the $x_{r}$ coordinate) of $B_{r}(n)$.

\section{Equivalence Between Translates and Scaled Copies}

\subsection{Applications of the Equivalence}

Before we present the proof of Theorem 2, we show some of its applications. In particular, we bound the maximum number of scaled copies of an arithmetic progression. Recall that $A_{k}=\{1,2,3, \ldots, k\}$. Clearly $s_{A_{1}}(n)=n$. Under the equivalence given by Theorem 2, the real patterns $A_{2}$ and $A_{3}$ correspond to the patterns $P_{1}=\{0,1\}$ and $P_{2}=\{(0,0),(1,0),(0,1)\}$, respectively. Thus, by Theorem $1, s_{A_{2}}(n)=t_{P_{1}}(n)=$ $n-m_{1}(n)=n-1$ and $s_{A_{3}}(n)=t_{P_{2}}(n)=n-m_{2}(n)=n-\left\lceil\sqrt{2 n+\frac{1}{4}}-\frac{1}{2}\right\rceil$ (the last identity is an elementary calculation). Similarly, $A_{4}$ corresponds to the pattern $L=\{(0,0),(1,0),(2,0),(0,1)\}$, and thus $s_{A_{4}}(n)=t_{L}(n)=n-\lceil 2 \sqrt{n}-1\rceil$ by Theorem 6 (see Sect. 5). For larger arithmetic progressions, we do not have exact values of $s_{A_{k}}(n)$. Yet, we know its asymptotic behavior in terms of the rank of $\left\langle A_{k}\right\rangle$ as a multiplicative subgroup of $\mathbb{R}^{+}$. For example, if $k=5$, then $A_{5}$ has rank 3 (it actually corresponds to the pattern $\{(0,0,0),(1,0,0),(2,0,0),(0,1,0),(0,0,1)\})$. Then by (1), $s_{A_{5}}(n)=n-\Theta\left(n^{2 / 3}\right)$. In general, using the Fundamental Theorem of Arithmetic, any element of $A_{k}$ can be multiplicatively generated by the primes within $A_{k}$, and all these primes are necessary to generate $A_{k}$. That is, the rank of $A_{k}$ is equal to $\pi(k)$, the number of primes that are less than or equal to $k$. Thus by (1), $s_{A_{k}}(n)=n-\Theta\left(n^{1-1 / \pi(k)}\right)$, proving Theorem 3 . 
The values of $s_{R}(n)$ can be determined for other patterns. For example, if $R=$ $\{1,2,3,6\}$, then $R$ corresponds, under Theorem 2 , to $\square=\{(0,0),(1,0),(1,1),(0,1)\}$. By Theorem 6, $s_{R}(n)=t_{\square}(n)=n-\lceil 2 \sqrt{n}-1\rceil$. Other patterns like $R=\{1, \sqrt{2}, \pi\}$ correspond to the same pattern $P_{2}$ as $A_{3}$ (above), and thus $s_{R}(n)=n-$ $\left\lceil\sqrt{2 n+\frac{1}{4}}-\frac{1}{2}\right\rceil$.

\subsection{Proof of Theorem 2}

Let $R$ be a finite set in $\mathbb{R}^{+}$. By dilating $R$, we can assume that $1 \in R$. Recall that $\langle R\rangle$ is the multiplicative subgroup of $\mathbb{R}^{+}$generated by $R$. Let $\left\{x_{1}, x_{2}, \ldots, x_{q}\right\}$ be a basis of $\langle R\rangle$. Then $q$ is the rank of $\langle R\rangle$, and $\langle R\rangle=\left\{x_{1}^{k_{1}} x_{2}^{k_{2}} \cdots x_{q}^{k_{q}}: k_{i} \in \mathbb{Z}\right\}$. Since any scaled copy $\lambda R$ of $R$ is contained in a single equivalence class $\lambda\langle R\rangle \in \mathbb{R}^{+} /\langle R\rangle$, we can restrict the maximum for the function $s_{R}(n)$ to $\langle R\rangle$. The following lemma is the multiplicative analogue of Lemma 1.

Lemma 3 For any finite set $R \subseteq \mathbb{R}^{+}$,

$$
s_{R}(n)=\max \left\{s_{R}(Y): Y \subseteq\langle R\rangle,|Y|=n\right\} .
$$

Proof Take $Y \subseteq \mathbb{R}^{+}$and assume that $Y \supseteq Y_{1} \cup Y_{2}$, where $Y_{1}$ and $Y_{2}$ are contained in the different classes $\lambda_{1}\langle R\rangle$ and $\lambda_{2}\langle R\rangle$, respectively. For $k \in \mathbb{Z}$ large enough, the set $Y_{2}^{\prime}=\left(\lambda_{1} \lambda_{2}^{-1} x_{1}^{k}\right) Y_{2} \subseteq \lambda_{1}\langle R\rangle$ has no elements in common with $Y$, and $Y_{2}^{\prime}$ has as many scaled copies of $R$ as $Y_{2}$. Replacing $Y_{2}$ by $Y_{2}^{\prime}$ in $Y$ gives a set intersecting a fewer number of equivalence classes in $\mathbb{R}^{+} /\langle R\rangle$ and with at least as many scaled copies of $R$ as $Y$. Now, if $Y$ is contained in a single class $\lambda_{1}\langle R\rangle \in \mathbb{R}^{+} /\langle R\rangle$, the set $\left(\lambda_{1}^{-1}\right) Y \subseteq\langle R\rangle$ contains as many scaled copies of $R$ as $Y$, proving the identity.

The function $G:\langle R\rangle \rightarrow \mathbb{Z}^{q}$ given by $G\left(x_{1}^{k_{1}} x_{2}^{k_{2}} \ldots x_{q}^{k_{q}}\right)=\left(k_{1}, k_{2}, \ldots, k_{q}\right)$ is a group isomorphism. Thus $G(\lambda R)=G(\lambda)+G(R)$ for any $\lambda \in\langle R\rangle$, and $v+G(R)=$ $G\left(G^{-1}(v) R\right)$ for any $v \in \mathbb{Z}^{q}$. That is, scaled copies of $R$ in $\langle R\rangle \subseteq \mathbb{R}^{+}$uniquely correspond to translates of $G(R)$ in $\mathbb{Z}^{q} \subseteq \mathbb{R}^{q}$ under $G$. Hence,

$$
Y \subseteq\langle R\rangle \text { implies }|Y|=|G(Y)| \text { and } \quad s_{R}(Y)=t_{G(R)}(G(Y)) .
$$

Let $P$ be a finite set in $\mathbb{R}^{d}$ containing 0 and with rational affine dimension $r$. Assume that $\left\{v_{1}, v_{2}, \ldots, v_{r}\right\}$ is a basis of $\langle P\rangle_{+}$and consider any $r$ distinct primes $p_{1}, p_{2}, \ldots, p_{r}$. Then $\left\langle p_{1}, p_{2}, \ldots, p_{r}\right\rangle=\left\{p_{1}^{k_{1}} p_{2}^{k_{2}} \cdots p_{r}^{k_{r}}: k_{i} \in \mathbb{Z}\right\}$ is a multiplicative subgroup of $\mathbb{R}^{+}$, and the function $F:\langle P\rangle_{+} \rightarrow\left\langle p_{1}, p_{2}, \ldots, p_{r}\right\rangle$ given by $F\left(k_{1} v_{1}+k_{2} v_{2}+\cdots+k_{r} v_{r}\right)=p_{1}^{k_{1}} p_{2}^{k_{2}} \cdots p_{r}^{k_{r}}$ is a group isomorphism. Thus $F(v+$ $P)=F(v) F(P)$ for any $v \in\langle P\rangle_{+}$, and $\lambda F(P)=F\left(F^{-1}(\lambda)+P\right)$ for any $\lambda \in$ $\left\langle p_{1}, p_{2}, \ldots, p_{r}\right\rangle$. That is, translates of $P$ in $\langle P\rangle_{+} \subseteq \mathbb{R}^{d}$ uniquely correspond to scaled copies of $F(P)$ in $\left\langle p_{1}, p_{2}, \ldots, p_{r}\right\rangle \subseteq \mathbb{R}^{+}$. Hence,

$$
X \subseteq\langle P\rangle_{+} \text {implies }|X|=|F(X)| \text { and } t_{P}(X)=s_{F(P)}(F(X)) \text {. }
$$


By Lemma 3, (5), and Lemma 1 we have

$$
\begin{aligned}
s_{R}(n) & =\max \left\{s_{R}(Y): Y \subseteq\langle R\rangle,|Y|=n\right\} \\
& =\max \left\{t_{G(R)}(G(Y)): Y \subseteq\langle R\rangle,|Y|=n\right\} \\
& =\max \left\{t_{G(R)}(X): X \subseteq G(\langle R\rangle),|X|=n\right\}=t_{G(R)}(n) .
\end{aligned}
$$

To obtain the last identity, observe that $\langle G(R)\rangle_{+} \subseteq G(\langle R\rangle) \subseteq \mathbb{R}^{q}$. Lemma 1 guarantees that the maximum of $t_{G(R)}(X)$ over all $X$ in a subset of $\mathbb{R}^{q}$ containing $\langle G(R)\rangle_{+}$is exactly $t_{G(R)}(n)$. Moreover, since $G$ is an isomorphism and $1 \in R$, $G(1)=0 \in G(R)$ and $q=\operatorname{dim}_{\mathbb{Q}} G(R)$.

Similarly, by Lemma 1, (6), and Lemma 3, together with $\langle F(P)\rangle \subseteq F\left(\langle P\rangle_{+}\right) \subseteq$ $\mathbb{R}^{+}$, we have that

$$
\begin{aligned}
t_{P}(n) & =\max \left\{t_{P}(X): X \subseteq\langle P\rangle_{+},|X|=n\right\} \\
& =\max \left\{s_{F(P)}(F(X)): X \subseteq\langle P\rangle_{+},|X|=n\right\} \\
& =\max \left\{s_{F(P)}(Y): Y \subseteq F\left(\langle P\rangle_{+}\right),|Y|=n\right\}=s_{F(P)}(n) .
\end{aligned}
$$

Since $F$ is an isomorphism and $0 \in P$, we have that $F(0)=1 \in F(P)$ and $r$ is the rank of $P$. Finally, observe that for a suitable $\lambda>0$, we can guarantee that $\lambda F(P) \subseteq$ $\mathbb{N}$, so we may assume that $F(P) \subseteq \mathbb{N}$.

\section{One-Dimensional Translated Patterns}

Let $P \subseteq \mathbb{R}^{d}$ be a pattern with $\operatorname{dim}_{\mathbb{Q}}(P)=1$. Then all members of $P$ are rational multiples of a vector $z \in \mathbb{R}^{d}$. Thus, using a suitable affine transformation, we can assume that $P=\left\{0, a_{1}, a_{2}, \ldots, a_{k}, a_{k+1}\right\} \subseteq \mathbb{Z}$ with $0<a_{1}<a_{2}<\cdots<a_{k}<a_{k+1}=$ $M$ and $\operatorname{gcd}\left\{a_{i}\right\}_{i=1}^{k+1}=1$. Clearly, if $|P|=1$, then $t_{P}(n)=n$. If $|P|=2$, then we can assume that $P=\{0,1\}$. By Theorem $1, t_{P}(n)=n-m_{1}(n)=n-1$. Theorem 4 shows that $t_{P}(n)=n-M$ for $|P| \geq 3$ and $n$ large enough, namely $n \geq M(M-k)$.

\subsection{Proof of Theorem 4}

Let $A_{n}=\{1,2,3, \ldots, n\}$. If $n \geq M$, clearly $x+P \subseteq A_{n}$ if and only if $1 \leq x \leq n-M$. Thus, $t_{P}(n) \geq t_{P}\left(A_{n}\right)=n-M$.

Now we prove that $t_{P}(n) \leq n-M$ for any $n$-set $X$ and $n \geq M(M-k)$. We study the subset of $X$ consisting of the first points of translates of $P$. Specifically, let $X_{0}=$ $\{x \in X: x+P \subseteq X\}$. Clearly $\left|X_{0}\right|=t_{P}(X)$. We consider the residue classes of $X_{0}$ modulo $M$. For $1 \leq i \leq M$, let $B_{i}=\left\{x \in X_{0}: x+i \equiv 0(\bmod M)\right\}, C_{i}=\left\{x \in X \backslash X_{0}\right.$ : $x+i \equiv 0(\bmod M)\}$, and set $x_{i}=\left|B_{i}\right|, y_{i}=\left|C_{i}\right|$. Of course,

$$
\sum_{i=1}^{M}\left(x_{i}+y_{i}\right)=n \quad \text { and } \quad \sum_{i=1}^{M} x_{i}=t_{P}(X) .
$$


Now observe that if $B_{i} \neq \varnothing$ and $m_{i}=\max B_{i}$, then $m_{i}+M \in C_{i} \neq \varnothing$, i.e.,

$$
y_{i}=0 \quad \text { implies } \quad x_{i}=0 .
$$

By definition, if $x \in B_{i} \subseteq X_{0}$, then $x+a_{j}+\left(i-a_{j}\right) \equiv x+i \equiv 0(\bmod M)$ and $x+a_{j} \in X$. Thus, $B_{i}+a_{j} \subseteq B_{i-a_{j}} \cup C_{i-a_{j}}$, that is, $x_{i} \leq x_{i-a_{j}}+y_{i-a_{j}}$. Replacing $i$ by $i+a_{j}$, we get

$$
x_{i+a_{j}}-x_{i} \leq y_{i} \quad \text { for } 0 \leq i \leq M-1 \text { and } 1 \leq j \leq k,
$$

where the indices are taken $\bmod M$.

Let $I=\left\{i: y_{i}=0\right\}$. If $I=\varnothing$, then $t_{P}(X)=\sum_{i=1}^{M} x_{i}=n-\sum_{i=1}^{M} y_{i} \leq n-M$ by (7). If $I \neq \varnothing$, we may assume, using a suitable translation of $X$, that $y_{M}=0$. Then by (8) $x_{M}=x_{0}=0$ and by (9) $x_{a_{j}}=0$ for all $j$. Let $A=\left(a_{1}, a_{2}, \ldots, a_{k}\right)$; for every $N=\left(n_{1}, n_{2}, \ldots, n_{k}\right) \in\left(\mathbb{N}^{*}\right)^{k}$, we define the dot product $N \cdot A=\sum_{j=1}^{k} n_{j} a_{j}$ and the norm $|N|=\sum_{j=1}^{k} n_{j}$. We also define a partial order on the vectors in $\left(\mathbb{N}^{*}\right)^{k}$ : If $N, N^{\prime} \in\left(\mathbb{N}^{*}\right)^{k}$, we say that $N^{\prime} \prec N$ if $n_{i}^{\prime} \leq n_{i}$ for $1 \leq i \leq k$ and $N^{\prime} \neq N$. We claim the following:

Claim For every $1 \leq u<M$, there is a vector $N=\left(n_{1}, n_{2}, \ldots, n_{k}\right) \in\left(\mathbb{N}^{*}\right)^{k}$ such that $N \cdot A \equiv u(\bmod M)$ and $N_{1} \cdot A \not \equiv N_{2} \cdot A(\bmod M)$ for every $N_{1} \prec N_{2} \preceq N$.

Proof First, since $\operatorname{gcd}\left\{a_{i}\right\}_{i=1}^{k+1}=1$, the set of vectors $N \in\left(\mathbb{N}^{*}\right)^{k}$ such that $N \cdot A \equiv$ $u(\bmod M)$ is nonempty. Suppose that $N \in\left(\mathbb{N}^{*}\right)^{k}$ satisfies $N \cdot A \equiv u(\bmod M)$ and minimizes $|N|$. Clearly, if $N_{1} \prec N$, then $\left|N_{1}\right|<|N|$ and consequently $N_{1} \cdot A \not \equiv$ $u(\bmod M)$. This proves the result when $N_{2}=N$. Similarly, if $0 \prec N^{\prime} \prec N$, then $N^{\prime} \cdot A \not \equiv 0(\bmod M)$. Otherwise $\left|N-N^{\prime}\right|<|N|$ and $\left(N-N^{\prime}\right) \cdot A \equiv u(\bmod M)$. Suppose that $N_{1} \prec N_{2} \preceq N$ and $N_{1} \cdot A \equiv N_{2} \cdot A(\bmod M)$. Then $0 \prec N_{2}-N_{1} \prec N$ and $\left(N_{2}-N_{1}\right) \cdot A \equiv 0(\bmod M)$, which contradicts the observation above. This proves the claim.

Let $N$ be a vector given by the claim. For every $1 \leq t \leq|N|$, we define $v(t) \in$ $\left(\mathbb{N}^{*}\right)^{k}$ as

$$
v(t)=\left(n_{1}, n_{2}, \ldots, n_{q_{t}}, r_{t}, 0, \ldots, 0\right),
$$

where $0 \leq q_{t} \leq k-1$ and $1 \leq r_{t} \leq n_{q_{t}+1}$ are the unique integers such that $|v(t)|=$ $n_{1}+\cdots+n_{q_{t}}+r_{t}=t$. By construction $0 \prec v(1) \prec v(2) \prec \cdots \prec v(|N|)=N$, and by the claim all residues $\bmod M$ of $v(t) \cdot A, 1 \leq t \leq|N|$ are pairwise different. By (9) we have for $1 \leq t \leq|N|-1$,

$$
x_{v(t+1) \cdot A}-x_{v(t) \cdot A} \leq y_{v(t) \cdot A} .
$$

Adding all these inequalities, since $x_{a_{1}}=0$, we obtain

$$
x_{u}=x_{u}-x_{a_{1}}=x_{N \cdot A}-x_{v(1) \cdot A} \leq \sum_{t=1}^{|N|-1} y_{v(t) \cdot A} \leq \sum_{j=0}^{M-1} y_{j}=n-t_{P}(X) .
$$


The last inequality follows from the fact that all the indices $v(t) \cdot A, 1 \leq t \leq|N|-1$ are pairwise different $\bmod M$. If we now add all these inequalities for every $u$ except $u=0, a_{1}, \ldots, a_{k}$ ( $x_{u}$ is equal to zero for these values), using (7), we get

$$
t_{P}(X)=\sum_{u=0}^{M-1} x_{u} \leq(M-k-1)\left(n-t_{P}(X)\right) .
$$

Finally, since $n \geq M(M-k)$, we have $t_{P}(X) \leq n-\frac{n}{M-k} \leq n-M$.

\subsection{Proof of Theorem 5}

Theorem 4 only works for $n$ large enough. Theorem 5 gives the exact value of $t_{P}(n)$ for all $n$ when $|P|=3$. In fact, Theorem 4 is implied (and improved) by Theorem 5 when $M$ is relatively prime to some $a_{i}, 1 \leq i \leq k$.

Let $P=\{0, a, b\} \subseteq \mathbb{Z}$ with $0<a<b$ and $\operatorname{gcd}(a, b)=1$. For $n \leq\left(\begin{array}{c}b \\ 2\end{array}\right)$, let $m$ and $p$ be the unique positive integers such that $n=\left(\begin{array}{c}m \\ 2\end{array}\right)+p$ with $1 \leq p \leq m$. A simple calculation shows that $m=\left\lceil\sqrt{2 n+\frac{1}{4}}-\frac{1}{2}\right\rceil$. Note that $1 \leq m \leq b-1$. We want to prove

$$
t_{P}(n)= \begin{cases}n-m & \text { if } n \leq\left(\begin{array}{c}
b \\
2
\end{array}\right), \\
n-b & \text { if } n>\left(\begin{array}{c}
b \\
2
\end{array}\right) .\end{cases}
$$

If $n \geq\left(\begin{array}{l}b \\ 2\end{array}\right)+1$, then $t_{P}(n) \geq t_{P}\left(A_{n}\right) \geq n-b$, as shown in the proof of Theorem 4 . If $n \leq\left(\begin{array}{l}b \\ 2\end{array}\right)$, we construct the $n$-set

$$
X_{n}=\bigcup_{j=0}^{m-1}\left\{a i+b j: 0 \leq i \leq m-j-2+\varepsilon_{j} \text { and } \varepsilon_{j}=\left\{\begin{array}{l}
1 \text { if } j \leq p-1 \\
0 \text { if } j \geq p
\end{array}\right\}\right.
$$

such that $t_{P}\left(X_{n}\right)=n-m$.

To check that $X_{n}$ has in fact $n$ elements, we show that the sets in the union above are pairwise disjoint, and thus $\left|X_{n}\right|=\sum_{j=0}^{m-1}(m-j-1)+\sum_{j=0}^{p-1} 1=\left(\begin{array}{c}m \\ 2\end{array}\right)+p=n$. Indeed, if $a i_{1}+b j_{1}=a i_{2}+b j_{2}$, then $a\left(i_{1}-i_{2}\right)=b\left(j_{1}-j_{2}\right)$, and since $\operatorname{gcd}(a, b)=1$, $i_{1} \equiv i_{2}(\bmod b)$. But $0 \leq i_{1}, i_{2}<m \leq b-1$, and thus $i_{1}=i_{2}$ and $j_{1}=j_{2}$.

To count the number of translates in $X_{n}$, define $X_{n}^{\prime}=\left\{x \in X_{n}: x+P \subseteq X_{n}\right\}$. By definition, $t_{P}\left(X_{n}\right)=\left|X_{n}^{\prime}\right|$. We claim that

$$
X_{n}^{\prime} \supseteq \bigcup_{j=0}^{m-2}\left\{a i+b j: 0 \leq i \leq m-j-3+\varepsilon_{j+1}\right\} .
$$

Indeed, suppose that $x=a i+b j$ with $0 \leq j \leq m-2$ and $0 \leq i \leq m-j-3+\varepsilon_{j+1}$. Note that $x \in X_{n}$. Since $\varepsilon_{j+1}=1$ implies $\varepsilon_{j}=1$, we have $x+a=a(i+1)+b j \in X_{n}$ and thus $1 \leq i+1 \leq m-j-2+\varepsilon_{j+1} \leq m-j-2+\varepsilon_{j}$. Similarly, $x+b=a i+$ $b(j+1) \in X_{n}$, since $0 \leq i \leq m-(j+1)-2+\varepsilon_{j+1}$. Finally,

$$
t_{P}\left(X_{n}\right)=\left|X_{n}^{\prime}\right| \geq \sum_{j=0}^{m-2}(m-j-2)+\sum_{j=0}^{p-2} 1=\left(\begin{array}{c}
m-1 \\
2
\end{array}\right)+p-1
$$




$$
=\left(\begin{array}{c}
m-1 \\
2
\end{array}\right)+n-\left(\begin{array}{c}
m \\
2
\end{array}\right)-1=n-m .
$$

Now, we argue that the right-hand side of (10) bounds $t_{P}(n)$ above. We follow the proof and notation of Theorem 4 with $k=1, a_{1}=a$, and $a_{2}=M=b$. But $B_{i}$ and $C_{i}$ are somewhat different: $B_{i}=\left\{x \in X_{0}: x+i a \equiv 0(\bmod b)\right\}$ and $C_{i}=\{x \in$ $\left.X \backslash X_{0}: x+i a \equiv 0(\bmod b)\right\}$ for $0 \leq i \leq b-1$. Again, let $x_{i}=\left|B_{i}\right|, y_{i}=\left|C_{i}\right|$, and $I=\left\{i: y_{i}=0\right\}$. In this case, (7) and (8) still hold, and (9) becomes

$$
x_{i}-x_{i-1} \leq y_{i-1},
$$

where all indices of $x$ and $y$ are taken $\bmod b$. Again, if $I=\varnothing$, then $t_{P}(X) \leq n-$ $b \leq n-m$. The same occurs if $s=\sum_{i=1}^{b} y_{i} \geq b$. From this point on, the proofs of Theorems 4 and 5 diverge.

Suppose now that $I \neq \varnothing, s<b$, and $\left\{x_{i}\right\},\left\{y_{i}\right\}$ are nonnegative integers maximizing $\sum_{i=1}^{b} x_{i}$ subject to the restrictions in (7), (8), and (11). If $y_{i}=0$, then by (8) $x_{i}=0$ and then by (11) $x_{i+1}=0$. Suppose that $1 \leq z(1)<z(2)<\cdots<z(k)$ are the members of $I$. By a suitable translation of $X$, we may assume that $z(1)=1$. Note that $1 \leq k<b \leq s$. Based on our previous observation and (11), we have for $1 \leq j \leq k$,

$$
\begin{aligned}
x_{z(j)} & =x_{z(j)+1}=0=y_{z(j)}, \\
x_{z(j)+2} & \leq y_{z(j)+1}, \\
x_{z(j)+3} & \leq y_{z(j)+1}+y_{z(j)+2}, \\
\vdots & \\
x_{z(j+1)-1} & \leq y_{z(j)+1}+y_{z(j)+2}+\cdots+y_{z(j+1)-2} .
\end{aligned}
$$

Thus,

$$
\sum_{i=0}^{b-1} x_{i} \leq \sum_{j=1}^{k} \sum_{u=0}^{z(j+1)-z(j)-1} y_{z(j)+u}(z(j+1)-z(j)-1-u) .
$$

(For convenience, we added zero-terms to the second sum when $u=0$ or $u=$ $z(j+1)-z(j)+1$. Also $z(k+1)=b+1$ by convention.) The following lemma allows us to bound the right-hand side of (12). The proof of the lemma consists of solving a simple linear programming problem. We defer the proof of this lemma to Sect. 4.3 and continue with the proof of the theorem.

Lemma 4 Let $b, k, s \in \mathbb{Z}^{+}$be such that $1 \leq k<b \leq s$. Consider the function

$$
G\left(y_{1}, y_{2}, \ldots, y_{b}\right)=\sum_{j=1}^{k} \sum_{u=0}^{z(j+1)-z(j)-1} y_{z(j)+u}(z(j+1)-z(j)-1-u),
$$

where $y_{i} \in \mathbb{N}^{*}, I=\left\{i: y_{i}=0\right\}=\{z(1), z(2), \ldots, z(k)\}$ with $1=z(1)<z(2)<\cdots<$ $z(k)\left(z(k+1)=b+1\right.$ by convention), and $s=\sum_{i=1}^{b} y_{i}$.

$G$ is maximized (uniquely except for symmetries $\bmod b$ ) by $y_{1}=y_{2}=\cdots=y_{k}=$ $0, y_{k+1}=s-(b-k-1)$, and $y_{k+2}=y_{k+3}=\cdots=y_{b}=1$. 
By Lemma 4, we can assume that $y_{1}=y_{2}=\cdots=y_{k}=0, y_{k+1}=s-(b-k-1)$, and $y_{k+2}=y_{k+3}=\cdots=y_{b}=1$. Then,

$$
\begin{aligned}
t_{P}(X) & =\sum_{i=0}^{b-1} x_{i} \leq \sum_{u=1}^{b-k-1} y_{k+u}(b-k-u) \\
& \leq(s-(b-k-1))(b-k-1)+\left(\begin{array}{c}
b-k-1 \\
2
\end{array}\right)=s(b-k-1)-\left(\begin{array}{c}
b-k \\
2
\end{array}\right) .
\end{aligned}
$$

Now recall that $s=n-t_{P}(X)$. Thus,

$$
t_{P}(X) \leq n-\left(\frac{n}{b-k}+\frac{b-k-1}{2}\right) .
$$

Let $l=m$ if $n \leq\left(\begin{array}{l}b \\ 2\end{array}\right)$ and $l=b$ otherwise. Note that $n>\left(\begin{array}{l}l \\ 2\end{array}\right)$. Since $k \in \mathbb{Z}$, we have

$$
\begin{aligned}
(b-k)(l-1)-\left(\begin{array}{c}
b-k \\
2
\end{array}\right) & =\frac{1}{2}\left(l-\frac{1}{2}\right)^{2}-\frac{1}{2}\left(b-k-\left(l-\frac{1}{2}\right)\right)^{2} \\
& \leq \frac{1}{2}\left(\left(l-\frac{1}{2}\right)^{2}-\frac{1}{4}\right)=\left(\begin{array}{l}
l \\
2
\end{array}\right) .
\end{aligned}
$$

Divide by $b-k$ to get $l-1 \leq\left(\begin{array}{l}l \\ 2\end{array}\right) /(b-k)+(b-k-1) / 2$, and thus

$$
t_{P}(X)<n-\left(\frac{\left(\begin{array}{l}
l \\
2
\end{array}\right)}{b-k}+\frac{b-k-1}{2}\right) \leq n-(l-1),
$$

that is, $t_{P}(X) \leq n-l$, and this completes the proof of the theorem.

\subsection{Proof of Lemma 4}

We first establish the following:

Claim Let $l \geq 1$ be an integer and $z>l$ a real number. The expression $x_{2}+2 x_{3}+$ $3 x_{4}+\cdots+l x_{l+1}$ subject to the conditions $x_{i} \geq 1$ and $x_{1}+x_{2}+\cdots+x_{l+1}=z$ is maximized uniquely by $x_{1}=x_{2}=\cdots=x_{l}=1$ and $x_{l+1}=z-l$.

Proof If $x_{i}>1$ for some $i \leq l$, then let $x_{l+1}^{\prime}=x_{l+1}+1, x_{i}^{\prime}=x_{i}-1$, and $x_{j}^{\prime}=x_{j}$ for all other indices. Then $\left(x_{2}^{\prime}+2 x_{3}^{\prime}+3 x_{4}^{\prime}+\cdots+l x_{l+1}^{\prime}\right)-\left(x_{2}+2 x_{3}+3 x_{4}+\cdots+\right.$ $\left.l x_{l+1}\right)=l\left(x_{l+1}+1\right)+(i-1)\left(x_{i}-1\right)-l x_{l+1}-(i-1) x_{i}=l-i+1 \geq 1$, which proves the claim.

Suppose $y$ maximizes $G(y)$. By using a suitable rotation $\bmod b$ we can assume that $y_{b} \neq 0$. Let $s_{j}=\sum_{i=z(j)}^{z(j+1)} y_{i}$. If $z(j+1)-z(j) \geq 2$, then, by the last claim, the $j$ th sum in (13) is maximized uniquely when $y_{z(j)+1}=s_{j}-(z(j+1)-z(j)-$ 2) and $y_{z(j)+2}=y_{z(j)+3}=\cdots=y_{z(j+1)-1}=1$. For the sake of brevity, let $d_{j}=$ $z(j+1)-z(j)$. Then the $j$ th sum in (13) equals

$$
\left(s_{j}-d_{j}+2\right)\left(d_{j}-2\right)+\left(\begin{array}{c}
d_{j}-2 \\
2
\end{array}\right)=\left(s_{j}\left(d_{j}-2\right)-\left(\begin{array}{c}
d_{j}-1 \\
2
\end{array}\right)\right) .
$$


If $z(j+1)-z(j)=1$, then the $j$ th sum in (13) is zero. Note that this agrees with the right-hand side of the previous equality. Thus we may assume that

$$
G(y)=\sum_{j=1}^{k}\left(s_{j}\left(d_{j}-2\right)-\left(\begin{array}{c}
d_{j}-1 \\
2
\end{array}\right)\right) .
$$

Now suppose that $s_{j}>0$ for some $j<k$. Note that $s_{j} \geq z(j+1)-z(j)-1=d_{j}-$ $1 \geq 1$. We will show that this is not the case where $y$ maximizes $G(y)$ by "moving" the $(j+1)$ th zero next to the $j$ th zero. Let $y_{z(j)+1}^{\prime}=0, y_{z(j)+2}^{\prime}=s(j)+s(j+1)-$ $(z(j+2)-z(j)-3)$ and $y_{z(j)+3}^{\prime}=y_{z(j)+4}^{\prime} \cdots=y_{z(j+2)-1}^{\prime}=1$. All other coordinates of $y^{\prime}$ are the same as those of $y$. Then $s_{j}^{\prime}=0, s_{j+1}^{\prime}=s_{j}+s_{j+1}$, and $s_{i}^{\prime}=s_{i}$ for all $i \neq j, j+1$. Also $z^{\prime}(j+1)=z(j)+1$ and $z^{\prime}(i)=z(i)$ for all $i \neq j+1$, i.e., $d_{j}^{\prime}=1$, $d_{j+1}^{\prime}=d_{j+1}+d_{j}-1$, and $d_{i}^{\prime}=d_{i}$ for all $i \neq j, j+1$. Thus,

$$
\begin{aligned}
G\left(y^{\prime}\right)-G(y)= & s_{j+1}^{\prime}\left(d_{j+1}^{\prime}-2\right)-\left(\begin{array}{c}
d_{j+1}^{\prime}-1 \\
2
\end{array}\right) \\
& -\left(s_{j}\left(d_{j}-2\right)-\left(\begin{array}{c}
d_{j}-1 \\
2
\end{array}\right)+s_{j+1}\left(d_{j+1}-2\right)-\left(\begin{array}{c}
d_{j+1}-1 \\
2
\end{array}\right)\right) \\
= & \left(s_{j}+s_{j+1}\right)\left(d_{j+1}+d_{j}-3\right)-\left(\begin{array}{c}
d_{j+1}+d_{j}-2 \\
2
\end{array}\right) \\
& \quad+\left(\begin{array}{c}
d_{j+1}-1 \\
2
\end{array}\right)+\left(\begin{array}{c}
d_{j}-1 \\
2
\end{array}\right)-s_{j}\left(d_{j}-2\right)-s_{j+1}\left(d_{j+1}-2\right) \\
= & \left(d_{j+1}-1\right)\left(s_{j}-\left(d_{j}-1\right)\right)+s_{j+1}\left(d_{j}-1\right) \\
\geq & s_{j+1}\left(d_{j}-1\right) \geq s_{j+1} \geq 0 .
\end{aligned}
$$

Moreover, unless all the $k$ zeros are the first $k$ integers, repeating this operation will eventually yield a strict inequality (one with $s_{j+1}>0$ ) because $y_{b} \neq 0$. This completes the proof of the lemma.

\section{Four-Point Sets with Rational Dimension Two}

If $P$ is a 3-point pattern, then an exact formula for $t_{P}(n)$ follows from Theorem 1 if $\operatorname{dim}_{\mathbb{Q}} P=2$ or from Theorem 5 if $\operatorname{dim}_{\mathbb{Q}} P=1$. For sets $P$ with 4 points, we also have exact formulas for $t_{P}(n)$ when $P$ has rational dimension 3 or 1 (at least for $n$ large enough). The only remaining case is when $\operatorname{dim}_{\mathbb{Q}} P=2$. In what follows, we show formulas for two particular patterns and present some conjectures for the general situation.

\subsection{The Square and the $L$}

First we present exact results for two important patterns. In particular the pattern $L$ below is equivalent, under Theorem 2, to the four-term arithmetic progression $A_{4}$. Thus, $s_{A_{4}}(n)=t_{L}(n)$. 


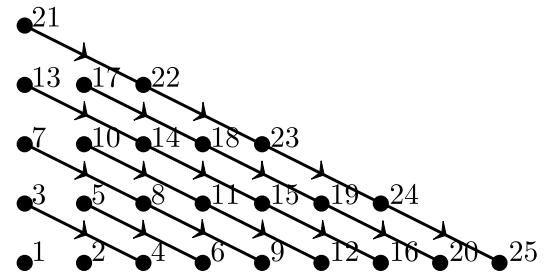

(a)

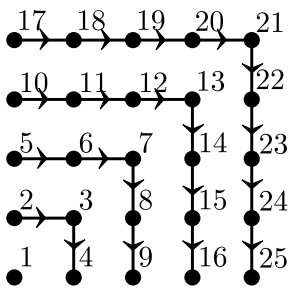

(b)

Fig. 4 Constructions of optimal sets $X_{25}$ and $Y_{25}$

Theorem 6 Let $L=\{(0,0),(1,0),(2,0),(0,1)\}$ and $\square=\{(0,0),(1,0),(0,1)$, $(1,1)\}$. Then for every $n \in \mathbb{N}, t_{L}(n)=t \square(n)=n-\lceil 2 \sqrt{n}-1\rceil$.

Proof For the constructive lower bounds, see Fig. 4(a) for the set $L$ and Fig. 4(b) for the set $\square$. For both $L$ and $\square$, the respective optimal sets $X_{n}$ and $Y_{n}$ consist of the points with labels 1 to $n$. The labels are assigned by following the pattern shown in the figure. It can be verified that $t_{L}\left(X_{n}\right)=t_{\square}\left(Y_{n}\right)=n-\lceil 2 \sqrt{n}-1\rceil$. We omit the details.

We now prove both upper bounds. The proof follows the ideas and notation of Theorem 1. Let $P \in\{L, \square\}$ and suppose that $X$ is an $n$-set with $t_{P}(X)=t_{P}(n)$. As in the proof of Theorem 1 , we can assume that $X \subseteq \mathbb{Z}^{2}$, all $y$-coordinates of $X$ are positive, and some are equal to one. We also use the notation $d_{P}(X)$ to denote the number of deficit points of $X$ with respect to $P, L_{i}$ to denote the points in $X$ with $y$-coordinate equal to $i$, and $\left|L_{i}\right|=l_{i}$. Note that $l_{1} \geq 1$.

We first consider $P=L$. Assume that $l_{1} \geq 2 \sqrt{n}-1$. Every non-deficit point $x \in$ $L_{i}$ is bijectively associated to $x+(0,1) \in L_{i+1}$. Thus there are at least $l_{i}-l_{i+1}$ deficit points in $L_{i}$ and then $d_{L}(X) \geq l_{1} \geq 2 \sqrt{n}-1$. Now assume that $l_{1}<2 \sqrt{n}-1$. If $l_{1}=1$, then the point in $l_{1}$ is clearly a deficit point, so $d_{L}(X) \geq 1+d_{L}\left(X \backslash L_{1}\right) \geq$ $1+d_{L}(n-1)$. By induction, $1+d_{L}(n-1) \geq 2 \sqrt{n-1}$, which is at least $2 \sqrt{n}-1$ for $n \geq 2$. If $l_{1} \geq 2$, then the last two points in $L_{1}$ are deficit points. In that case, $d_{L}(X) \geq$ $2+d_{L}\left(X \backslash L_{1}\right) \geq 2+d_{L}\left(n-l_{1}\right)$. By induction, $2+d_{L}\left(n-l_{1}\right) \geq 2+2 \sqrt{n-l_{1}}-$ 1 , which is greater than $2 \sqrt{n}-1$ as long as $l_{1}<2 \sqrt{n}-1$. In all cases, $t_{L}(n)=$ $n-d_{L}(n) \leq n-\lceil 2 \sqrt{n}-1\rceil$.

Let $P=\square$ and proceed by induction on $n$. Suppose that $u$ is the largest number for which $l_{u} \geq 1$. If $l_{j}=0$ for some $1<j<u$, then the result follows by induction applied to $L_{1} \cup \cdots \cup L_{j-1}$ and $L_{j+1} \cup \cdots \cup L_{u}$ (the function $2 \sqrt{n}-1$ is concave down). Assume that $l_{j} \geq 1$ for all $1 \leq j \leq u$. We have

$$
d_{\square}(X) \geq \sum_{i=1}^{u-1} \max \left(1, l_{i}-l_{i+1}+1\right)+l_{u} .
$$

Indeed, every last point in $L_{i}$ is a deficit point with respect to $\square$. Also, if $i<u$, there are at most $l_{i+1}-1$ points $x$ in $L_{i+1}$ which are not deficit points with respect to $\{(0,0),(1,0)\}$. Then there are at least $l_{i}-\left(l_{i+1}-1\right)=l_{i}-l_{i+1}+1$ points in $L_{i}$ 
which are not exactly below any of these points $x$, and thus they are deficit points with respect to $\square$. Finally, all points in $L_{u}$ are clearly deficit points.

Since $\max \left(1, l_{i}-l_{i+1}+1\right)=\left(2+l_{i}-l_{i+1}+\left|l_{i}-l_{i+1}\right|\right) / 2$, using the conventions $l_{0}=l_{u+1}=0$, we have

$$
\begin{aligned}
d_{\square}(X) & \geq \frac{1}{2} \sum_{i=1}^{u-1}\left(2+l_{i}-l_{i+1}+\left|l_{i}-l_{i+1}\right|\right)+l_{u} \\
& =u-1+\frac{l_{1}}{2}+\frac{l_{u}}{2}+\frac{1}{2} \sum_{i=1}^{u-1}\left|l_{i}-l_{i+1}\right| \\
& \geq u-1+\frac{1}{2} \sum_{i=0}^{u}\left|l_{i}-l_{i+1}\right| \\
& \geq u-1+\max _{1 \leq i \leq u} l_{i} \quad(\text { by the triangle inequality }) \\
& \geq u-1+\frac{1}{u} \sum_{i=1}^{u} l_{i}=u+\frac{n}{u}-1 \geq 2 \sqrt{n}-1 .
\end{aligned}
$$

Thus, $t_{\square}(n)=n-d_{\square}(n) \leq n-\lceil 2 \sqrt{n}-1\rceil$.

\subsection{Conjectures for Arbitrary 4-Point Sets $P$}

Now we discuss a plan and some conjectures to completely determine the function $t_{P}(n)$ when $P$ is a four-point-set of affine rational dimension two. First note that, under an affine transformation $T$, translates of $P$ correspond bijectively to translates of $T(P)$. Since $P$ has rational dimension two, we can assume that $P=$ $\{(0,0),(1,0),(0,1),(x, y)\}$, where $x, y \in \mathbb{Q}$. Moreover, by choosing appropriately the point in $P$ corresponding to $(x, y)$, we can assume that $(x, y)$ is not in the interior or the boundary of the triangle $(0,0),(1,0),(0,1)$. That is, we can assume that $x<0$ or $y<0$ or $x+y \geq 1$.

Let $z=(x, y) \in \mathbb{Q}^{2}$. We can uniquely write $x=p_{x} / q$ and $y=p_{y} / q$, where $p_{x}, p_{y}, q$ are integers, $q>0$, and $\operatorname{gcd}\left(p_{x}, p_{y}, q\right)=1$. Define $F: \mathbb{Q}^{2} \rightarrow \mathbb{N}$ as

$$
F(z)=F(x, y)= \begin{cases}p_{x}+p_{y} & \text { if } p_{x}, p_{y} \geq 0 \\ \max \left(p_{x},-p_{y}+q\right) & \text { if } p_{y}<0 \leq p_{x} \\ \max \left(p_{y},-p_{x}+q\right) & \text { if } p_{x}<0 \leq p_{y} \\ -p_{x}-p_{y}+q & \text { if } p_{x}, p_{y}<0\end{cases}
$$

Let $\Omega=\left\{(x, y) \in \mathbb{Q}^{2}: x<0\right.$ or $y<0$ or $\left.x+y \geq 1\right\}$. For every $z=(x, y) \in \Omega$, let $P(z)=\{(0,0),(1,0),(0,1),(x, y)\}$. We conjecture that:

Conjecture 1 If $z_{1}, z_{2} \in \Omega$ and $F\left(z_{1}\right)=F\left(z_{2}\right)$, then $t_{P\left(z_{1}\right)}(n)=t_{P\left(z_{2}\right)}(n)$ for every $n \in \mathbb{N}$. 

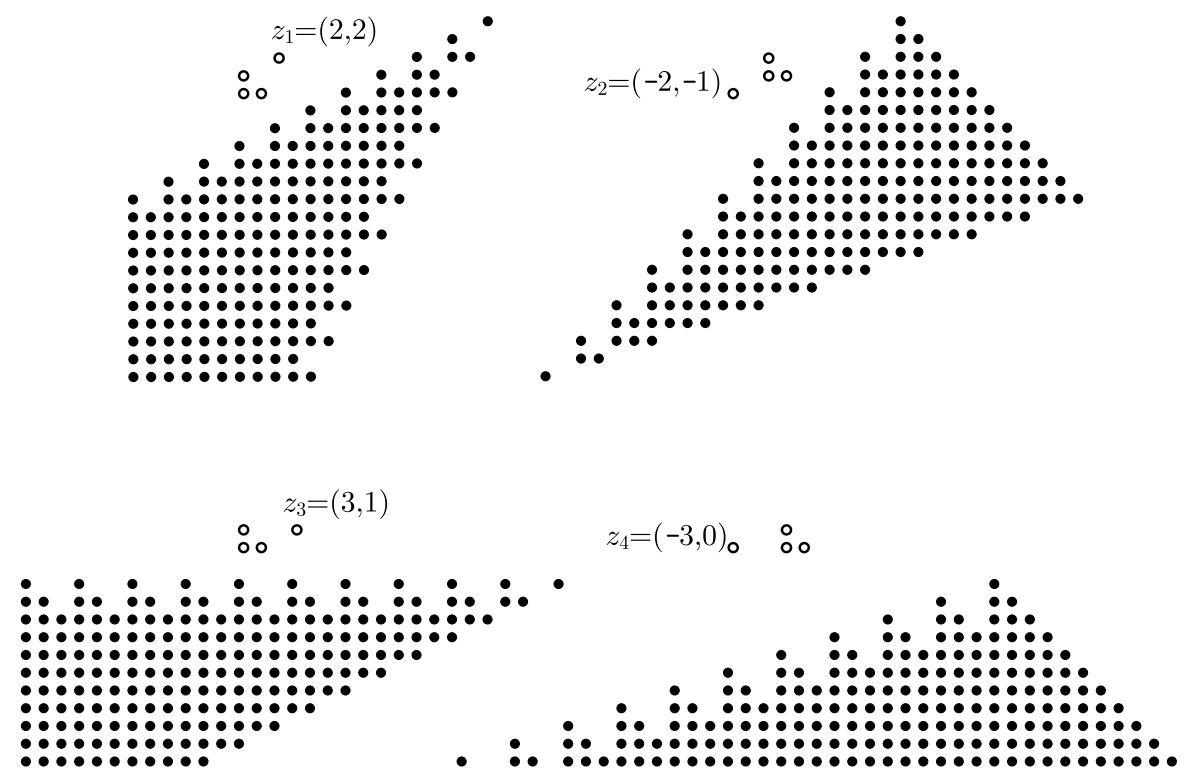

Fig. 5 Conjectured optimal constructions for the functions $t_{P\left(z_{i}\right)}(n)$, where $n=102$

The previous section shows how this conjecture is true when $F(z)=2$. Figure 5 shows four sets $P\left(z_{i}\right), 1 \leq i \leq 4$, for which $F\left(z_{i}\right)=4$ and the conjectured optimal constructions for $n=102$. In fact, every other $z \in \Omega$ with $F(z)=4$ satisfies that $P(z)$ is affinely equivalent to one of the sets in Fig. 5. If Conjecture 1 is true in general, then it would be enough to determine $t_{P(z)}(n)$ for $z$ of the form $z=(a, 0)$ with $a \in \mathbb{N}$. By considering these particular sets, we further believe that the correct coefficient for the $n^{1 / 2}$ term is $\sqrt{2 F(z)}$ and that the rest of the error term is constant. More precisely:

Conjecture 2 For every $z \in \Omega$,

$$
t_{P(z)}(n)=n-\sqrt{2 F(z)} n^{1 / 2}+O(1) .
$$

For every set $P(z)$ with $z \in \Omega$, we can construct $n$-element sets $X_{n}$ with $n-\sqrt{2 F(z)} n^{1 / 2}+O(1)$ translates of $P(z)$. However we were able to prove a matching upper bound only for sets of the form $z=(a, 0)$. For instance, we can prove $t_{P(3,0)}(n)=n-\lceil\sqrt{6 n-15 / 4}-3 / 2\rceil$ for $n \geq 4$ and, in general, $t_{P(a, 0)}(n) \leq$ $n-\left\lceil\sqrt{2 a n-a^{2}\left(a^{2}-4\right) / 12}-a / 2\right\rceil$ for $a \geq 2$ and $n \geq\left(\begin{array}{c}a+1 \\ 3\end{array}\right)$. Already for $a=4$ we cannot match, for every $n$, the $O(1)$ terms from our construction and from the previous upper bound. It is likely that an exact formula will depend on some kind of binomial-type representation, much like Theorem 1.

Acknowledgements We are grateful to E. Rivera-Campo for constructive discussions and suggestions. 


\section{References}

1. Ábrego, B.M., Elekes, Gy., Fernández-Merchant, S.: Structural results for planar sets with many similar subsets. Combinatorica 24, 541-554 (2004)

2. Ábrego, B.M., Fernández-Merchant, S., Llano, B.: An inequality for Kruskal-Macaulay functions. arXiv:0809.3549v1 (2007)

3. Anderson, I.: Combinatorics of finite sets. Dover, Mineola (2002), ISBN 0-486-42257-7, 145 pp. (Corrected reprint of the 1989 edition published by Oxford University Press, Oxford)

4. Brass, P.: Combinatorial geometry problems in pattern recognition. Discrete Comput. Geom. 28, 495510 (2002)

5. Brass, P.: On the maximum number of unit distances among $n$ points in dimension four. In: Bárány, I., et al. (eds.) Intuitive Geometry. Bolyai Soc. Mathematical Studies, vol. 6, pp. 277-290 (1997)

6. Brass, P., Pach, J.: Problems and results on geometric patterns. In: Avis, D. et al. (eds.) Graph Theory and Combinatorial Optimization, pp. 17-36. Springer, New York (2005)

7. Brass, P., Moser, W., Pach, J.: Research Problems in Discrete Geometry. Springer, New York (2005)

8. Elekes, G., Erdős, P.: Similar configurations and pseudogrids. In: Böröczky, K., et al. (eds.) Intuitive Geometry (Szeged, 1991), Colloq. Math. Soc. János Bolyai, vol. 63, pp. 85-104 (1994)

9. Erdős, P.: On sets of distances of $n$ points. Am. Math. Mon. 53, 248-250 (1946)

10. Erdős, P., Purdy, G.: Some extremal problems in geometry. J. Comb. Theory Ser. A 10, 246-252 (1971)

11. Erdős, P., Purdy, G.: Some extremal problems in geometry IV. Congres. Numer. 17, 307-322 (1976) (Proc. 7th South-Eastern Conf. Combinatorics, Graph Theory, and Computing)

12. Knuth, D.E.: The Art of Computer Programming, vol. 4, Fascicle 3, Generating All Combinations and Partitions (2005), vi + 150 pp. ISBN 0-201-85394-5

13. Laczkovich, M., Ruzsa, I.Z.: The number of homothetic subsets. In: Graham, R.L. et al. (eds.) The Mathematics of Paul Erdős vol. II, Algorithms and Combinatorics Ser. 14, pp. 294-302. Springer, New York (1997)

14. Macaulay, F.S.: Some properties of enumeration in theory of modular systems. Proc. Lond. Math. Soc. 26, 531-555 (1927)

15. Pach, J., Sharir, M.: Combinatorial Geometry with Algorithmic Applications, The Alcala Lectures, Alcala, Spain, August 31-September 5 (2006). http://www.cmr.es/Publications/quaderns/notes39.pdf or http://www.math.tau.il/ michas/notes.pdf

16. Swanepoel, K.: Unit distances and diameters in Euclidean spaces. Discrete Comput. Geom. (2008). doi:10.1007/s00454-008-9082-X

17. van Wamelen, P.: The maximum number of unit distances among $n$ points in dimension four. Beitr. Algebra Geom. 40, 475-477 (1999) 